\title{
Longitudinal associations between ultra-processed foods and blood lipids in childhood
}

Paula S. Leffa ${ }^{1 *}$, Daniel J. Hoffman ${ }^{2}$, Fernanda Rauber ${ }^{3,4}$, Caroline N. Sangalli ${ }^{1}$, Júlia L. Valmórbida ${ }^{5}$ and Márcia R. Vitolo ${ }^{1,5}$

${ }^{1}$ Graduate Program of Health Sciences, Universidade Federal de Ciências da Saúde de Porto Alegre, Porto Alegre, Brazil ${ }^{2}$ Department of Nutritional Sciences, New Jersey Institute for Food, Nutrition, and Health, Center for Childhood Nutrition Research, Rutgers, the State University of New Jersey, New Brunswick, NJ, USA

${ }^{3}$ Department of Nutrition, School of Public Health, University of São Paulo, São Paulo, Brazil

${ }^{4}$ Center for Epidemiological Research in Nutrition and Health, University of São Paulo, São Paulo, Brazil

${ }^{5}$ Graduate Program in Pediatrics: Child and Adolescent Health Care, Universidade Federal de Ciências da Saúde de Porto Alegre, Porto Alegre, Brazil

(Submitted 13 January 2020 - Final revision received 17 March 2020 - Accepted 25 March 2020 - First published online 6 April 2020)

\section{Abstract}

Emerging evidence suggests that the consumption of ultra-processed foods (UPF) plays a role in the development of chronic diseases, but evidence of their influence in children is limited. Our objective was to study longitudinal trends of UPF intake and determine their impact on blood lipids in young children. The present study was a follow-up of a randomised field trial of children ( $n$ 308) from Porto Alegre, Brazil. Dietary intake was collected using two 24-h recalls at 3 and 6 years of age, and consumption of UPF was classified according to the NOVA system, a food classification based on the extent and purpose of industrial food processing. At age 6 years, blood tests were performed to measure lipid profile. Contribution of UPF to total energy intake increased by $10 \%$ during the follow-up period, from $43.4 \%$ at 3 years to $47.7 \%$ at 6 years of age. Linear regression models showed that children in the highest tertile of UPF consumption at age 3 years had higher levels of total cholesterol (TC; $\beta 0.22 \mathrm{mmol} / \mathrm{l} ; 95 \% \mathrm{CI} 0 \cdot 04,0 \cdot 39)$ and TAG at age 6 years ( $\beta 0 \cdot 11 \mathrm{mmol} / 1,95 \% \mathrm{CI} 0 \cdot 01,0 \cdot 20)$ compared with those in the lowest tertile. A positive dose-response was observed for an absolute increment of $10 \%$ of UPF on TC ( $\beta$ 0.07 mmol/1, 95\% CI 0.00, 0.14) and TAG ( $\beta 0.04 \mathrm{mmol} / \mathrm{l}, 95 \% \mathrm{CI} 0 \cdot 01,0.07)$. Based on our data, consumption of UPF increased significantly over time and was associated with higher blood lipid levels in children from a low-income community. Our findings highlight the need for effective strategies to minimise the consumption of UPF in early life.

Key words: Ultra-processed food: Children: Trends: Lipid profile: Longitudinal studies

CVD, a leading cause of the global burden of death and disease in adults ${ }^{(1,2)}$, is attributed to a number of predisposing factors including low physical activity and an unhealthy dietary pattern $^{(3,4)}$. Recent research suggests that a major contributor of unhealthy dietary patterns is a high intake of ultra-processed foods (UPF) ${ }^{(5)}$. Moreover, it has been reported that risk factors driving CVD, such as high serum lipid concentrations, have their origins even in early childhood ${ }^{(6,7)}$. High serum lipid profiles are directly affected by a poor diet, despite the fact that diet is one of the most important modifiable risk factors in the prevention of chronic non-communicable diseases ${ }^{(8-10)}$. However, an area that has received limited attention relative to UPF intake and lipid profiles in childhood is the focus of this paper.

The global consumption of UPF, characterised as readyto-eat and energy-dense manufactured foods, has increased dramatically ${ }^{(11-14)}$. Sales of UPF in low- and middle-income countries are rising at a disproportionate rate compared with high-income countries ${ }^{(15)}$. For example, Euromonitor reported a sales growth in UPF by $30 \%$ in Brazil from 2000 to 2013, while in the same period, sales dropped in the USA and Canada (-9 and $-7 \cdot 3 \%$, respectively $)^{(16)}$. Several studies have shown that high contribution of UPF in dietary patterns is associated with diets of lower nutritional quality ${ }^{(17-19)}$ assessed by NOVA, a classification of foods based on their degree and purpose of industrial food processing. These findings are especially important given that dietary patterns in this childhood often track into adulthood ${ }^{(20)}$.

Several studies have been conducted to quantify the burden of disease attributable to specific UPF consumption. In adults, consumption of UPF has been associated with an increased risk of overweight and obesity ${ }^{(21-23)}$, and related conditions, such as

Abbreviations: TC, total cholesterol; UPF, ultra-processed foods.

* Corresponding author: Paula S. Leffa, email paulaleffa@hotmail.com 
hypertension ${ }^{(24)}, \mathrm{CVD}^{(25)}$, as well as higher risk of overall cancer ${ }^{(26)}$. However, few studies focus on the association between consumption of UPF and risk of diseases in childhood $^{(27-29)}$. It is critical to better understand the possible effects of these changing patterns, in particular how they may influence metabolic risk factors for non-communicable diseases in the paediatric population. Therefore, the objective of our longitudinal study was to assess trends of UPF consumption and determine the association between UPF and lipid profile in a population of children in southern Brazil.

\section{Methods}

\section{Study design}

This longitudinal study used data from children at 3 and 6 years of age who participated in a randomised intervention study (NCT00635453) of breast-feeding and dietary practices ${ }^{(30)}$. The intervention trial was conducted in health centres that provide primary care services predominantly to low-income families in Porto Alegre, Brazil, and the outcomes were assessed for mothers and children who received treatment at these centres. Briefly, health workers (physicians, nurses and administrative staff) of all intervention health centres participated in a training based on the "Ten Steps for Healthy Feeding for Brazilian Children from Birth to Two Years of Age' guideline and strategies to provide suggestions how best to incorporate recommendations into the consultations. From April to December 2008, all pregnant women in the last trimester at the participating health centres were invited to sign up for outcome tracking by interviewers who were blinded to the allocation status of the participants. All births occurred between May 2008 and February 2009.

The sample size initially chosen for the trial was based on the goal of detecting a difference in the prevalence of exclusive breast-feeding at 4 months ${ }^{(31)}$. A power of $90 \%, \alpha$ of 0.5 , design effect of 1.5 and a loss prediction of $20 \%$ were used to calculate the sample size, resulting in the inclusion of 360 mother-baby pairs in the intervention group and another 355 in the control group. Of 715 pregnant women who registered initially, 635 of their children were enrolled at the study at 6 months of age. A total of 476 and 387 children at 3 and 6 years of age, respectively, underwent assessment in the follow-up study. As the present study had a different aim, we proceeded to estimate if the available sample at age 6 years was sufficient to investigate the association between UPF consumption and outcomes. Assuming a correlation coefficient in the order of 0.3 and a design effect of 2, a total sample size of 226 children was required (with $90 \%$ power and $\alpha=5 \%$ ). This study was conducted according to the guidelines laid down in the Declaration of Helsinki, and all procedures involving human subjects were approved by the Ethics Committee of the Universidade Federal de Ciências da Saúde de Porto Alegre. Informed consent was obtained from mothers on behalf of their children at each stage of data collection.

\section{Data collection}

Mothers were interviewed at home visits by trained interviewers when their children were at 6 months, 3 years and 6 years of age.
Home visits for data collection by the interviewers were verified through telephone callbacks to a $5 \%$ random sample of interviewed mothers. Socio-economic and family characteristics were obtained at recruitment. Birth weight and length, and sex were collected from the children's health records. Prepregnancy weight was self-reported, and mothers' height was measured during home visits when the children were 6 months of age and pre-pregnancy BMI was calculated. Anthropometric data of all children were obtained at 3 and 6 years using a digital scale to the nearest $0 \cdot 1 \mathrm{~kg}$ and a stadiometer to the nearest $0 \cdot 1 \mathrm{~cm}$. BMI for age $z$-score was calculated based on the WHO standards ${ }^{(32)}$, and obesity was defined as BMI $z$-score $>2$ sD for all ages.

\section{Dietary assessment}

Two multiple-pass 24-h dietary recalls were collected for each child at ages 3 and 6 years during the home visits on two nonconsecutive days that were chosen randomly within 2 weeks to 1 month $^{(33)}$. For children at age 3 years, recalls were provided by mothers or other caregivers; at age 6 years, children reported all the foods and beverages consumed the day before the interview, with help from their parents. For children who spent time with a caregiver other than the parent (e.g. during school hours), we contacted the caregiver to record all items the children consumed during the previous day. When it was not possible to obtain all available information, the participants were contacted on a different day to administer that 24-h recalls to provide accurate data for all children. Details about food types, amounts and preparation methods were recorded. Common household measures (e.g. teaspoons, tablespoons, cups and serving sizes) were used to help mothers report the amounts of food given to their children and to standardise portion sizes. Interviews were conducted by dietitians/nutritionists and undergraduate students in nutritional sciences trained and supported in the 24-h recall method with standardised procedures, including practice interviews prior to the start of the study. The research supervisor reviewed all the dietary recalls. Dietary energy intake was estimated using the Dietwin ${ }^{\circledR}$ software programme (version 2008 professional Dietwin ${ }^{\circledR}$ ), and the Brazilian Food Composition Table (TACO, 2006) was preferentially used as a reference, followed by the United States Department of Agriculture chemical composition tables (Agricultural Research Service, 1998). For commercial products, we manually added all nutritional composition provided by the manufacturer to the programme.

\section{Ultra-processed food consumption}

UPF was assessed using the NOVA classification system ${ }^{(34,35)}$, a four-group food classification based on the extent and purpose of food processing, including unprocessed and minimally processed foods, processed culinary ingredients, processed foods and UPF. This study focused on the NOVA group of UPF. Briefly, UPF are formulations of ingredients, most of exclusive industrial use, that result from a series of industrial processes and typically including little or no fresh food. UPF are ready to eat, drink or heat (e.g. soft drinks, sweet or savoury packaged snacks, breakfast cereal, candies, chocolate, 'instant' soups and noodles, processed meats, pre-prepared frozen dishes; and 
many other products). All food and drink items assessed in the dietary surveys were categorised as ultra-processed or not based on the food classification previously reported. This categorisation was performed by a team of dietitians trained, supervised by researchers. Home-made recipes were identified and decomposed using standardised recipes, and the classification was applied to their ingredients. For a small number of specific food items such as pizza, there was insufficient information for classification purposes. In those cases, we used a conservative approach, such that the lower level of processing was chosen. UPF subcategories analysed in the present study included (1) savoury and biscuits (crackers, chips, salty snacks, cookies), (2) soft drinks (soda, sweetened juice), (3) sweets (candies, chocolate and ice cream), (4) powdered chocolate, (5) sugary milk beverages, (6) processed meats, (7) breads, (8) baby cereal, (9) margarine, mayonnaise and dressing and (10) ready-made soup/noodle (instant noodle and dehydrated soup).

\section{Lipid profile}

Venous blood samples were collected at 6 years of age to assess serum lipid profile, and analyses were performed at the laboratory of the Universidade Federal de Ciências da Saúde de Porto Alegre. Total cholesterol (TC), HDL-cholesterol and TAG levels were measured using standard enzymatic methods with an automatic analyzer (BS-120, Mindray). LDL-cholesterol was calculated according to Friedewald's formula ${ }^{(36)}$. Cut-off values for abnormal lipid concentrations were defined according to the American College of Cardiology/American Heart Association (TC $\geq 4.40 \mathrm{mmol} / \mathrm{l}, \mathrm{LDL} \geq 2.85 \mathrm{mmol} / \mathrm{l}, \mathrm{HDL}<1.17 \mathrm{mmol} / 1$ and $\mathrm{TG} \geq 0.85 \mathrm{mmol} / \mathrm{l}^{(37)}$.

\section{Data analysis}

The usual dietary energy intake of UPF was estimated by the Multiple Source Method (https://msm.dife.de) ${ }^{(38)}$ that calculates dietary intake for individuals and then constructs the population distribution based on these data ${ }^{(39,40)}$. All participants were considered consumers of total energy intake and for UPF groups; a probability value of $0.5(50 \%)$ was used to assign the status of habitual consumer. Contribution of each subgroup of UPF to the total energy intake was calculated as a percentage of total energy.

Differences between intervention and control groups with respect to UPF consumption were analysed, and no differences at $3(P=0.697)$ and $6(P=0.606)$ years of age were observed. Continuous variables were expressed as mean and standard deviation (normally distributed data) or median and interquartile range (non-normally distributed data) and percentage frequency. Analyses of trends in UPF consumption from age 3 to 6 years were conducted using Wilcoxon signed pair test, and the percentage change across study period was evaluated.

Crude and multivariable linear regression models were used to determine the relationship between consumption of UPF at age 3 years and lipid profiles at age 6 years. All models were adjusted for sex, group status in early phase (intervention or control), birth weight, pre-pregnancy BMI and family income (model 1), in addition to BMI $z$-score at baseline (3 years) (model 2) and for total energy intake and percentage of fat intake at age 3 years (model 3 ). The lowest tertile was used as the reference group, and differences between strata of UPF consumption are presented as standardised regression coefficients with $95 \%$ CI. We also estimated the change in lipid levels for a $10 \%$ increase in UPF consumption (continuous variable). Tests of linear trend were performed in all models by tertiles of UPF consumption as a continuous variable. All statistical analyses were conducted using SPSS version 21.0 (IBM Statistics Inc.), and statistical significance was set at $P<0.05$.

\section{Results}

Of the 633 originally infants at 6 months of age included in the study, 476 and 387 children underwent assessment at the mean age of 3 and 6 years, respectively. The main reasons for loss were refusal to participate in the study and change of address (Fig. 1). No differences were found for sex, birth weight, maternal age at child's birth and family income between the children who were lost to follow-up and those who remained at 6 years of age $(P>0.05$; data not shown). The final analysis included 308 children with both complete dietary at age 3 years and blood data at age 6 years.

Characteristics of the study participants are presented in Table 1 . Briefly, $18.9 \%$ of the mothers were under age 20 years at the child's birth and $28.9 \%$ had 8 years of schooling or less. Family income was low for most families $-62.5 \%$ had a monthly income less than three times the national minimum wage (approximately $\$ 804 \cdot 00 /$ month). More than half of the children were male $(52.0 \%)$, and the obesity prevalence was $18 \%$ at age 3 years and $18.4 \%$ at age 6 years. Descriptive information of the lipid profile is shown in Table 2. Among children at age 6 years, prevalence of abnormal concentrations of TC, TAG, LDL-cholesterol and HDL-cholesterol was 39.9, 36.0, 14.0, and $8.8 \%$, respectively.

Consumption of UPF represented a median of 43.4 and $47.7 \%$ of the total energy intake at 3 and 6 years of age, respectively (Table 3). Within food groups, the significant energy contributors were savoury and biscuits, soft drinks and sweets in both age groups. Taken together, these UPF provided 25.8 and $28.4 \%$ of the total energy intake at age 3 and 6 years, respectively. The overall dietary contribution of UPF increased by $10 \%$ $(P<0 \cdot 001)$ during a mean follow-up period of 3 years. The largest relative increase among UPF groups occurred for margarine, mayonnaise and dressing (1200\%; $P<0.001)$. There were notable changes in processed meat $(58.3 \% ; P<0.001)$ and bread $(19.3 \% ; \quad P<0.001)$ consumption during childhood. Decreasing intake was seen for sugary milk beverages $(41.0 \% ; \quad P<0.001)$ and powdered chocolate $(27.5 \%$ $P<0 \cdot 001)$ over the study period.

Longitudinal associations between UPF intake and lipid profile are shown in Table 4. There was a positive association between UPF consumption at age 3 years and total serum cholesterol at age 6 years. In the fully adjusted model, higher UPF intake was associated with increased levels of total serum cholesterol later at age 6 years (tertile $3 v$. tertile $1 ; \beta 0.22 \mathrm{mmol} / \mathrm{l}$; $95 \%$ CI 0.04, 0.39). Similarly, a $10 \%$ increase in the consumption of UPF was associated with a $0.07 \mathrm{mmol} / 1$ (95\% CI $0 \cdot 00,0 \cdot 14)$ 


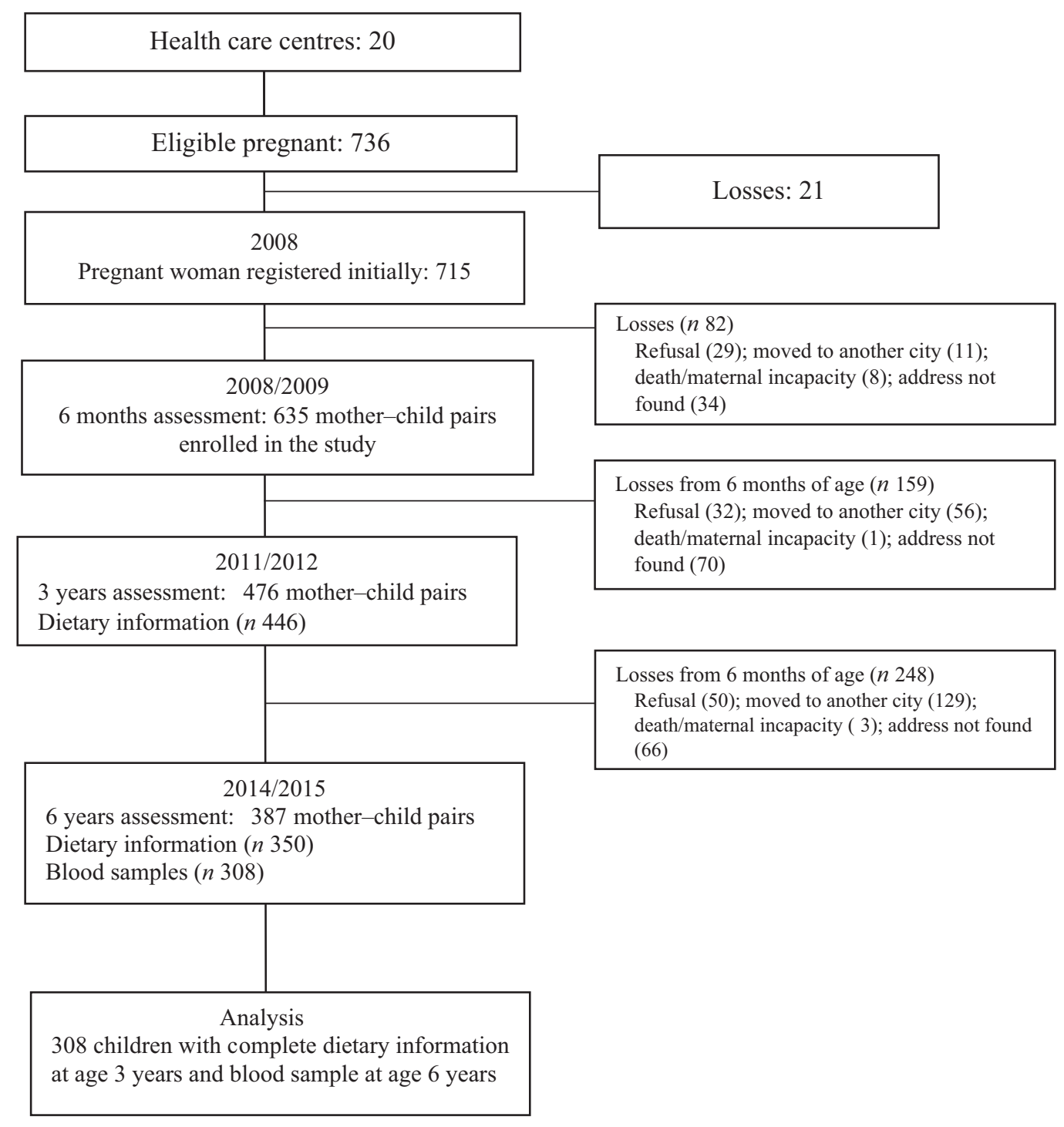

Fig. 1. Flow diagram of the study.

increase in TC. Children in the highest tertile of UPF consumption had a mean TAG value of $0 \cdot 11 \mathrm{mmol} / 1$ higher $(95 \% \mathrm{CI} 0 \cdot 01$, $0 \cdot 20)$ than those in the lowest tertile consumption in the fully adjusted model. Statistically significant associations were observed for an absolute increment of 10 in the percentage of UPF and TAG ( $\beta 0.04 \mathrm{mmol} / 1,95 \%$ CI 0.01, 0.07).

\section{Discussion}

While the global consumption of UPF has increased greatly, only a few studies have reported an association between UPF intake and health outcomes in children ${ }^{(27-29)}$. Results from our longitudinal study suggest that higher UPF consumption during childhood was associated with higher levels of TC and TAG. In addition, we found that the consumption of UPF increased greatly during this period of early childhood. Overall, our results support the recent report of the FAO of the United Nations ${ }^{(5)}$ that highlights the need for studies analysing the impact of UPF as a risk factor for chronic non-communicable diseases in childhood.

Our data provide a comprehensive view of changes in UPF consumption from early to middle childhood. Briefly, UPF accounted for approximately $50 \%$ of total energy consumed by the children, even for those in the lowest tertile of UPF intake, the consumption of these products was particularly high in this population compared with the general Brazilian population ${ }^{(41)}$. We also found a $10 \%$ increase in total UPF consumption during the study period, suggesting a decline in diet quality the children studied. The increase in energy provided by the UPF is accompanied by a change in types of these products consumed, with the replacement of UPF targeting toddlers, such as 'sugary milk beverages' and 'baby cereals', by a dietary pattern based on ready-to-eat meals, such as sandwiches made with processed meat, bread and margarine, mayonnaise or dressing. Moreover, this shift in dietary patterns may be a result of complex social and environmental factors. For example, families in 
Table 1. Characteristics of children and their families $(n 308)^{*}$ (Numbers and percentages; mean values and standard deviations)

\begin{tabular}{|c|c|c|c|}
\hline Characteristics & Mean & & SD \\
\hline \multicolumn{4}{|c|}{ Maternal and family } \\
\hline \multicolumn{4}{|c|}{ Maternal pre-gestational BMI $\geq 25 \mathrm{~kg} / \mathrm{m}^{2}$} \\
\hline$n$ & & 131 & \\
\hline$\%$ & & 38.0 & \\
\hline \multicolumn{4}{|c|}{ Maternal age at child's birth $<20$ years } \\
\hline$n$ & & 66 & \\
\hline$\%$ & & 18.9 & \\
\hline \multicolumn{4}{|c|}{ Mother's education $\leq 8$ years } \\
\hline$n$ & & 101 & \\
\hline$\%$ & & 28.9 & \\
\hline \multicolumn{4}{|c|}{ Annual household income $<$ US $\$ 3000$} \\
\hline$n$ & & 55 & \\
\hline$\%$ & & $16 \cdot 0$ & \\
\hline \multicolumn{4}{|l|}{ Child at 3 years } \\
\hline Age (years) & $3 \cdot 2$ & & 0.1 \\
\hline Weight (kg) & $15 \cdot 8$ & & 2.5 \\
\hline Height $(\mathrm{cm})$ & 96.7 & & $4 \cdot 3$ \\
\hline BMI $z$-score & 0.9 & & $1 \cdot 2$ \\
\hline \multicolumn{4}{|l|}{ Obesity } \\
\hline$n$ & & 81 & \\
\hline$\%$ & & $18 \cdot 0$ & \\
\hline \multicolumn{4}{|l|}{ Child at 6 years } \\
\hline Age (years) & $6 \cdot 3$ & & 0.2 \\
\hline Weight (kg) & $24 \cdot 1$ & & $5 \cdot 6$ \\
\hline Height $(\mathrm{cm})$ & $119 \cdot 1$ & & 5.7 \\
\hline BMI $z$-score & 0.7 & & 1.5 \\
\hline \multicolumn{4}{|l|}{ Obesity } \\
\hline$n$ & & 58 & \\
\hline$\%$ & & 18.4 & \\
\hline
\end{tabular}

* Values may not equal to total number of subjects in each group because of missing data.

Table 2. Prevalence of alterations in lipid profile at age 6 years ( $n$ 308) (Numbers and percentages; mean values and standard deviations)

\begin{tabular}{lccrr}
\hline & & & \multicolumn{2}{c}{ Abnormal values } \\
\cline { 4 - 5 } Lipid profile $(\mathrm{mmol} / \mathrm{l})$ & Mean & SD & $n$ & $\%$ \\
\hline Total cholesterol & 4.20 & 0.69 & $123^{*}$ & 39.9 \\
LDL-cholesterol & 2.20 & 0.60 & $43 \dagger$ & 14.0 \\
HDL-cholesterol & 1.63 & 0.32 & $27 \ddagger$ & 8.8 \\
TAG & 0.80 & 0.34 & $111 \S$ & 36.0 \\
\hline${ }^{*} \geq 4.40 \mathrm{mmol} / \mathrm{l}$. & & & & \\
$\dagger \geq 2.85 \mathrm{mmol} / \mathrm{l}$. & & & & \\
$\ddagger<1.17 \mathrm{mmol} / \mathrm{l}$. & & & & \\
$\S \geq 0.85 \mathrm{mmol} / \mathrm{l}$. & & &
\end{tabular}

low-income urban neighbourhoods have limited availability of healthful food and a greater availability of $\mathrm{UPF}^{(42-44)}$. Likewise, low-income groups, such as families enrolled in this study, are more likely to choose low-cost foods when available ${ }^{(45)}$. Thus, providing detailed information about food consumption during childhood is necessary to develop effective strategies to prevent the chronic diseases later in life.

In regard to the development of chronic diseases, we found that higher consumption of UPF at age 3 years was significantly associated with higher blood lipid concentrations at age 6 years. There are a number of explanations for the results provided. For example, a high consumption of UPF is associated with unhealthful dietary patterns, characterised by an excess intake of energy, fat and added sugar ${ }^{(18,41,46)}$, especially among children and adolescents ${ }^{(47,48)}$. Indeed, increased total energy intake, which is mainly driven by overconsumption of high-fat and high-sugar foods, is associated with increased lipogenesis and increased concentrations of circulating TAG and cholesterol ${ }^{(3)}$. Moreover, it has been reported that an excess intake of dietary sugar may be a central mediator for de novo lipogenesis, stimulating overproduction of hepatic TAG, resulting in hypertriacylglycerolaemia ${ }^{(49,50)}$. In addition to nutrient level mechanisms, an excess intake of UPF is inversely associated with a lower intake of fruits and vegetables, foods that are known to prevent $\mathrm{CVD}^{(51)}$. Furthermore, given that obesity is a significant predictor of poor cardiometabolic health ${ }^{(52,53)}$, a high intake of UPF, which has occurred following aggressive advertising and marketing of $\mathrm{UPF}^{(54,55)}$, may promote obesity through a disruption of hunger and satiety ${ }^{(56,57)}$. Thus, these mechanisms support the association between UPF consumption and an unhealthy lipid profile, potentially increasing the risk of CVD.

Given the increased prevalence of CVD throughout the world $^{(1)}$, our findings may be used to better elucidate the impact of UPF consumption on risk factors for CVD. For example, elevated TC and TAG are of critical significance since these results suggest an early vascular inflammatory response $e^{(7,58)}$. As the intake of UPF can continue increasing in lifelong, there is a real potential that these products may later affect the metabolism of LDL- and HDL-cholesterol. It is worth noting that significant changes generally occur in lipoprotein metabolism following puberty ${ }^{(59)}$, which could explain the absence of association between UPF consumption and LDL- and HDL-cholesterol in our study. A cohort study in France reported that an increment of 10 in the percentage of UPF consumption was associated with a $12 \%$ increase in the rates of CVD in adults ${ }^{(25)}$. A longitudinal study of 8-year-old Brazilian children found an association between consumption of UPF and higher levels of total and LDL-cholesterol ${ }^{(27)}$, but not of higher TAG. Nonetheless, our results extend this work by studying younger children who lived in a major urban centre with greater access to UPF. The negative health risks of UPF consumption in the paediatric population are of particular concern as blood lipid profiles may worsen later in life as undesirable changes in eating behaviours are common during the transition from childhood to adolescence ${ }^{(60)}$. From a public health perspective, the robust evidence demonstrating the impact of UPF consumption on child health, investing in diet quality is one of the main priorities to promote cardiovascular health ${ }^{(61)}$.

There are potential limitations of our study that should be discussed to fully appreciate our results. First, a number of participants were lost during follow-up, but there were no significant differences between the baseline characteristics of children who remained in the study and those lost to follow-up. Second, cautious generalisation is required since the majority of our sample had low family income and may limit the applicability of our findings for more privileged populations. Third, the average of the two dietary recalls may not represent the entire distribution of usual intake due to the intra-individual variance component. Despite these limitations, our study has a number of strengths that merit attention. First, the prospective study design allowed us to assess the longitudinal association between UPF intake and lipid profile. Second, we collected detailed dietary data, including food preparation methods, ingredients used in dishes and the brand of commercial products, which allowed us to classify 
Table 3. Trends in the consumption of ultra-processed foods (UPF) (\% of total energy) at ages 3 and 6 years old (Median values and interquartile ranges (IQR))

\begin{tabular}{|c|c|c|c|c|c|c|}
\hline & \multicolumn{2}{|c|}{3 years } & \multicolumn{2}{|c|}{6 years } & \multirow[b]{2}{*}{$P_{\text {for trend }}$} & \multirow[b]{2}{*}{ Percentage change } \\
\hline & Median & IQR & Median & IQR & & \\
\hline \multicolumn{7}{|l|}{ UPF groups* } \\
\hline Savoury and biscuits $†$ & 9.7 & $4 \cdot 6-14 \cdot 0$ & 11.5 & $7 \cdot 1-16 \cdot 2$ & $<0.001$ & $18 \cdot 5$ \\
\hline Soft drinksł & $8 \cdot 1$ & $4 \cdot 7-10 \cdot 9$ & 8.2 & $5 \cdot 5-10 \cdot 3$ & 0.752 & $1 \cdot 2$ \\
\hline Sweets§ & 8.0 & $4 \cdot 7-11 \cdot 1$ & 8.7 & $4 \cdot 1-13 \cdot 4$ & 0.021 & 8.8 \\
\hline Powdered chocolate & $4 \cdot 0$ & $0.8-6.3$ & 2.9 & $0.8-4.8$ & $<0.001$ & $-27 \cdot 5$ \\
\hline Sugary milk beverages & 3.9 & $0 \cdot 3-6 \cdot 1$ & $2 \cdot 3$ & $0.0-3 \cdot 8$ & $<0.001$ & -41.0 \\
\hline Processed meat & $2 \cdot 4$ & $0.6-4.4$ & 3.8 & $1.9-5.6$ & $<0.001$ & $58 \cdot 3$ \\
\hline Bread & $3 \cdot 1$ & $0.0-3.7$ & 3.7 & $1 \cdot 5-5 \cdot 8$ & $<0.001$ & $19 \cdot 3$ \\
\hline Baby cereals & $1 \cdot 8$ & $0.0-2.7$ & $1 \cdot 3$ & $0.0-1.7$ & 0.651 & $-27 \cdot 7$ \\
\hline Margarine, mayonnaise and dressings & $0 \cdot 2$ & $0.1-0.3$ & $2 \cdot 6$ & $1 \cdot 1-3 \cdot 6$ & $<0.001$ & $1200 \cdot 0$ \\
\hline Ready-made soup/noodles & $1 \cdot 1$ & $0.0-1 \cdot 3$ & 1.5 & $0.1-1 \cdot 8$ & 0.067 & $36 \cdot 4$ \\
\hline Total UPF & 43.4 & $34 \cdot 3-51 \cdot 1$ & $47 \cdot 7$ & $41 \cdot 5-53 \cdot 8$ & $<0.001$ & $9 \cdot 9$ \\
\hline
\end{tabular}

* Expressed as percentage of total energy intake.

† Crackers, chips, salty snacks, cookies.

¥ Soda, sweetened juice.

$\S$ Candy, chocolate and ice cream.

Table 4. Linear regression analyses of the association of ultra-processed food (UPF) consumption at 3 years old with lipid profile at 6 years old ( $\beta$ Values and $95 \%$ confidence intervals)

\begin{tabular}{|c|c|c|c|c|c|c|c|c|c|}
\hline & & & & $\mathrm{F}$ cons & tion (\% of tota & ergy) & & & \\
\hline & Tertile 1 & & rtile 2 & & tile 3 & & & tinuous & \\
\hline & $29.6(5.1-38.2) \%$ & 44. & $3-48.9) \%$ & $56 \cdot 6$ & $.0-85.3) \%$ & & & increase & \\
\hline & Reference & $\beta$ & $95 \% \mathrm{Cl}$ & $\beta$ & $95 \% \mathrm{Cl}$ & $P_{\text {for trend }}$ & $\beta$ & $95 \% \mathrm{Cl}$ & $P$ \\
\hline Total cholest & $(\mathrm{mmol} / \mathrm{l})$ & & & & & & & & \\
\hline Crude & 0 & 0.19 & $0.01,0.38$ & $0.24^{*}$ & $0.07,0.41$ & 0.016 & 0.07 & $0.01,0.14$ & 0.017 \\
\hline Model $1 \dagger$ & 0 & 0.21 & $0.01,0.40$ & $0.25^{*}$ & $0.06,0.42$ & 0.013 & 0.08 & $0.02,0.15$ & 0.017 \\
\hline Model 2‡ & 0 & 0.20 & $-0.00,0.40$ & $0.22^{*}$ & $0.03,0.41$ & 0.032 & 0.07 & $0.00,0.15$ & 0.044 \\
\hline Model $3 \S$ & 0 & 0.19 & $-0.01,0.40$ & $0.22^{*}$ & $0.04,0.39$ & 0.033 & 0.07 & $0.00,0.14$ & 0.046 \\
\hline LDL-choleste & $\mathrm{mmol} / \mathrm{l})$ & & & & & & & & \\
\hline Crude & 0 & 0.09 & $-0.09,0.27$ & 0.12 & $-0.01,0.25$ & 0.226 & 0.04 & $-0.00,0.10$ & 0.151 \\
\hline Model $1 \dagger$ & 0 & 0.10 & $-0.08,0.30$ & 0.12 & $-0.03,0.27$ & 0.288 & 0.04 & $-0.00,0.11$ & 0.153 \\
\hline Model 2‡ & 0 & 0.09 & $-0.10,0.30$ & 0.09 & $-0.06,0.24$ & 0.463 & 0.03 & $-0.02,0.09$ & 0.322 \\
\hline Model $3 \S$ & 0 & 0.10 & $-0.10,0.31$ & 0.09 & $-0.06,0.23$ & 0.467 & 0.03 & $-0.03,0.09$ & 0.331 \\
\hline HDL-cholest & $(\mathrm{mmol} / \mathrm{l})$ & & & & & & & & \\
\hline Crude & 0 & 0.07 & $-0.20,0.16$ & 0.06 & $-0.04,0.17$ & 0.257 & 0.02 & $-0.01,0.05$ & 0.271 \\
\hline Model $1 \dagger$ & 0 & 0.05 & $-0.03,0.14$ & 0.05 & $-0.06,0.16$ & 0.444 & 0.02 & $-0.02,0.05$ & 0.396 \\
\hline Model 2‡ & 0 & 0.04 & $-0.03,0.12$ & 0.05 & $-0.05,0.16$ & 0.444 & 0.01 & $-0.01,0.06$ & 0.318 \\
\hline Model $3 \S$ & 0 & 0.04 & $-0.03,0.12$ & 0.05 & $-0.06,0.15$ & 0.424 & 0.01 & $-0.02,0.05$ & 0.334 \\
\hline TAG $(\mathrm{mmol} / \mathrm{l}$ & & & & & & & & & \\
\hline Crude & 0 & 0.01 & $-0.08,0.10$ & 0.05 & $-0.02,0.13$ & 0.394 & 0.02 & $-0.01,0.05$ & 0.157 \\
\hline Model $1 \dagger$ & 0 & 0.02 & $-0.05,0.10$ & 0.08 & $-0.01,0.18$ & 0.205 & 0.03 & $-0.00,0.06$ & 0.061 \\
\hline Model $2 \ddagger$ & 0 & 0.04 & $-0.04,0.13$ & $0.10^{*}$ & $0.00,0.20$ & 0.117 & 0.03 & $0.00,0.07$ & 0.034 \\
\hline Model $3 \S$ & 0 & 0.03 & $-0.05,0.11$ & $0.11^{*}$ & $0.01,0.20$ & 0.085 & 0.04 & $0.01,0.07$ & 0.024 \\
\hline
\end{tabular}

${ }^{*}$ Difference between the first and third tertile $(P<0.05)$.

† Model $1=$ adjusted for sex, group status in the early phase (intervention and control), family income, pre-pregnancy BMI and child birth weight.

$\ddagger$ Model $2=$ model $1+$ BMl $z$-scores at 3 years.

$\S$ Model $3=$ model $2+$ intakes of total energy and total fat $(\%)$ at 3 years.

foods according to the NOVA system, a valid tool for public health and nutrition research and policy ${ }^{(16,62,63)}$.

In conclusion, we found that there has been a significant increase in the percentage of energy intake from UPF during childhood for children from a low-income community in Brazil. More important, we determined that a higher consumption of UPF was associated with increased blood lipids for the children in this study. The results of our study improve the understanding of how UPF intake may contribute to poor diet quality in this critical period of life. Thus, there is an urgent need for double- and tripleduty actions focused on minimising the consumption of UPF in early life to reduce the risk of CVD later in life.

\section{Acknowledgements}

The authors would like to thank the healthcare workers and families who participated in the study. 
This work was supported by Brazilian Ministry of Health (no. $577 / 200$ ), Research Support Foundation of the State of Rio Grande do Sul (PPSUS/2006/1537-7) and Brazilian National Council for Scientific and Technological Development (no $14 / 2013-47731 / 2013-8$ ). Also, this study was supported by Coordination for the Improvement of Higher Education Personnel (PSL doctoral fellowship, no. 88881.362086/2019-01).

P. S. L. formulated the research question, analysed and interpreted the data, performed statistical analysis and wrote the manuscript. D. J. H. interpreted the data and statistical analysis and contributed to the drafting and critical revision of the manuscript. F. R. undertook data, interpreted data analysis and critically reviewed the manuscript. C. N. S. and J. L. V. contributed to the drafting and critical revision of the manuscript. M. R. V. designed and conducted the study, formulated the research question, interpreted the data and the statistical analysis and critically reviewed the article.

None of the authors has any conflicts of interest to declare.

\section{References}

1. Roth GA, Johnson C, Abajobir A, et al. (2017) Global, regional, and national burden of cardiovascular diseases for 10 causes, 1990 to 2015. J Am Coll Cardiol 70, 1-25.

2. Mendis S, Armstrong T, Bettcher D, et al. (2014) Global Status Report on Noncommunicable Diseases: 2014. Geneva: World Health Organization.

3. Siri-Tarino PW \& Krauss RM (2016) Diet, lipids, and cardiovascular disease. Curr Opin Lipidol 27, 323-328.

4. Vos MB, Kaar JL, Welsh JA, et al. (2017) Added sugars and cardiovascular disease risk in children: a scientific statement from the American Heart Association. Circulation 135, e1017-e1034.

5. Monteiro CA, Cannon G, Lawrence M, et al. (2019) Ultraprocessed Foods, Diet Quality, and Health Using the NOVA Classification System. Rome: FAO.

6. Juhola J, Magnussen CG, Viikari JSA, et al. (2011) Tracking of serum lipid levels, blood pressure, and body mass index from childhood to adulthood: the Cardiovascular Risk in Young Finns Study. J Pediatr 159, 584-590.

7. Steinberger J, Daniels SR, Hagberg N, et al. (2016) Cardiovascular health promotion in children: challenges and opportunities for 2020 and beyond: a scientific statement from the American Heart Association. Circulation 134, e236-255.

8. GBD 2017 Diet Collaborators (2019) Health effects of dietary risks in 195 countries, 1990-2017: a systematic analysis for the Global Burden of Disease Study 2017. Lancet 393, 1958-1972.

9. Micha R, Peñalvo JL, Cudhea F, et al. (2017) Association between dietary factors and mortality from heart disease, stroke, and type 2 diabetes in the United States. JAMA 317, 912-924.

10. de Oliveira Otto MC, Afshin A, Micha R, et al. (2016) The impact of dietary and metabolic risk factors on cardiovascular diseases and type 2 diabetes mortality in Brazil. PLOS ONE 11, e0151503.

11. Monteiro CA, Moubarac J-C, Levy RB, et al. (2018) Household availability of ultra-processed foods and obesity in nineteen European countries. Public Health Nutr 21, 18-26.

12. Juul F \& Hemmingsson E (2015) Trends in consumption of ultra-processed foods and obesity in Sweden between 1960 and 2010. Public Health Nutr 18, 3096-3107.
13. Marrón-Ponce JA, Tolentino-Mayo L, Hernández-F M, et al. (2018) Trends in ultra-processed food purchases from 1984 to 2016 in Mexican households. Nutrients 11, 45.

14. Monteiro CA, Levy RB, Claro RM, et al. (2011) Increasing consumption of ultra-processed foods and likely impact on human health: evidence from Brazil. Public Health Nutr 14, 5-13.

15. Vandevijvere S, Jaacks LM, Monteiro CA, et al. (2019) Global trends in ultraprocessed food and drink product sales and their association with adult body mass index trajectories. Obes Rev 20, 10-19.

16. Pan American Health Organization (2015) Ultra-Processed Food and Drink Products in Latin America: Trends, Impact on Obesity, Policy Implications. Washington, DC: PAHO. http://www.paho.org/hq/index.php?option=com_content\&view= article\&id $=11153 \% 3$ Aultra-processedfood-and-drinkproducts \&catid=4999\%Adocuments\&lang=en (accessed April 2020).

17. Louzada ML da C, Ricardo CZ, Steele EM, et al. (2018) The share of ultra-processed foods determines the overall nutritional quality of diets in Brazil. Public Health Nutr 21, 94-102.

18. Rauber F, da Costa Louzada ML, Steele EM, et al. (2018) Ultraprocessed food consumption and chronic non-communicable diseases-related dietary nutrient profile in the UK (2008-2014). Nutrients 10, 587.

19. Martínez Steele E, Popkin BM, Swinburn B, et al. (2017) The share of ultra-processed foods and the overall nutritional quality of diets in the US: evidence from a nationally representative cross-sectional study. Popul Health Metr 15, 6.

20. Ventura AK \& Worobey J (2013) Early influences on the development of food preferences. Curr Biol 23, R401-R408.

21. Mendonça R de D, Pimenta AM, Gea A, et al. (2016) Ultraprocessed food consumption and risk of overweight and obesity: the University of Navarra Follow-Up (SUN) cohort study. Am J Clin Nutr 104, 1433-1440.

22. Canhada SL, Luft VC, Giatti L, et al. (2020) Ultra-processed foods, incident overweight and obesity, and longitudinal changes in weight and waist circumference: the Brazilian Longitudinal Study of Adult Health (ELSA-Brasil). Public Health Nutr 23, 1076-1086

23. Juul F, Martinez-Steele E, Parekh $\mathrm{N}$, et al. (2018) Ultraprocessed food consumption and excess weight among US adults. Br J Nutr 120, 90-100.

24. Mendonça R de D, Lopes ACS, Pimenta AM, et al. (2017) Ultraprocessed food consumption and the incidence of hypertension in a Mediterranean cohort: the Seguimiento Universidad de Navarra Project. Am J Hypertens 30, 358-366.

25. Srour B, Fezeu LK, Kesse-Guyot E, et al. (2019) Ultra-processed food intake and risk of cardiovascular disease: prospective cohort study (NutriNet-Santé). BMJ 365, 11451.

26. Fiolet T, Srour B, Sellem L, et al. (2018) Consumption of ultraprocessed foods and cancer risk: results from NutriNet-Santé prospective cohort. BMJ 360, k322.

27. Rauber F, Campagnolo PDB, Hoffman DJ, et al. (2015) Consumption of ultra-processed food products and its effects on children's lipid profiles: a longitudinal study. Nutr Metab Cardiovasc Dis 25, 116-122.

28. Costa CS, Rauber F, Leffa PS, et al. (2019) Ultra-processed food consumption and its effects on anthropometric and glucose profile: a longitudinal study during childhood. Nutr Metab Cardiovasc Dis 29, 177-184.

29. Machado Azeredo C, Cortese M, Costa CDS, et al. (2020) Ultraprocessed food consumption during childhood and asthma in adolescence: data from the 2004 Pelotas birth cohort study. Pediatr Allergy Immunol 31, 27-37.

30. Vitolo MR, Louzada ML da C \& Rauber F (2014) Positive impact of child feeding training program for primary care health 
professionals: a cluster randomized field trial. Rev Bras Epidemiol 17, 873-886.

31. Vitolo MR, Bortolini GA, Feldens CA, et al. (2005) Impacts of the 10 steps to healthy feeding in infants: a randomized field trial. Cad Saude Publica 21, 1448-1457.

32. World Health Organization (2006) WHO Child Growth Standards: Length/Height-for-Age, Weight-for-Age, Weightfor-Length, Weight-for-Height and Body Mass Index-for-Age; Methods and Development. Geneva: WHO Press.

33. Food and Agriculture Organization of the United Nations (2018) Dietary Assessment: A Resource Guide to Method Selection and Application in Low Resource Settings. Rome, Italy: FAO.

34. Monteiro CA, Cannon G, Levy RB, et al. (2019) Ultra-processed foods: what they are and how to identify them. Public Health Nutr 22, 936-941.

35. Monteiro CA, Cannon G, Moubarac J-C, et al. (2018) The UN Decade of Nutrition, the NOVA food classification and the trouble with ultra-processing. Public Health Nutr 21, 5-17.

36. Friedewald WT, Levy RI \& Fredrickson DS (1972) Estimation of the concentration of low-density lipoprotein cholesterol in plasma, without use of the preparative ultracentrifuge. Clin Chem 18, 499-502.

37. Grundy SM, Stone NJ, Bailey AL, et al. (2019) 2018 AHA/ACC/ AACVPR/AAPA/ABC/ACPM/ADA/AGS/APhA/ASPC/NLA/ PCNA Guideline on the Management of Blood Cholesterol: Executive Summary: A Report of the American College of Cardiology/American Heart Association Task Force on Clinical Practice Guidelines. J Am Coll Cardiol 73, 3168-3209.

38. Harttig U, Haubrock J, Knüppel S, et al. (2011) The MSM program: web-based statistics package for estimating usual dietary intake using the Multiple Source Method. Eur J Clin Nutr 65 , Suppl. 1, S87-S91.

39. Laureano G, Torman V, Crispim S, et al. (2016) Comparison of the ISU, NCI, MSM, and SPADE methods for estimating usual intake: a Simulation Study of Nutrients Consumed Daily. Nutrients $\mathbf{8}, 166$

40. Haubrock J, Nöthlings U, Volatier J-L, et al. (2011) Estimating usual food intake distributions by using the multiple source method in the EPIC-Potsdam Calibration Study. J Nutr 141, 914-920.

41. Louzada ML da C, Martins APB, Canella DS, et al. (2015) Ultraprocessed foods and the nutritional dietary profile in Brazil. Rev Saúde Pública 49, 38.

42. Pitt E, Gallegos D, Comans T, et al. (2017) Exploring the influence of local food environments on food behaviours: a systematic review of qualitative literature. Public Health Nutr 20, 2393-2405.

43. Lake AA (2018) Neighbourhood food environments: food choice, foodscapes and planning for health. Proc Nutr Soc 77, 239-246.

44. Leite FHM, de Carvalho Cremm E, de Abreu DSC, et al. (2018) Association of neighbourhood food availability with the consumption of processed and ultra-processed food products by children in a city of Brazil: a multilevel analysis. Public Health Nutr 21, 189-200.

45. Darmon N \& Drewnowski A (2015) Contribution of food prices and diet cost to socioeconomic disparities in diet quality and health: a systematic review and analysis. Nutr Rev 73, 643-660.

46. Neri D, Martinez-Steele E, Monteiro CA, et al. (2019) Consumption of ultra-processed foods and its association with added sugar content in the diets of US children, NHANES 2009-2014. Pediatr Obes 14, e12563.

47. Rauber F, Louzada ML da C, Martinez Steele E, et al. (2019) Ultra-processed foods and excessive free sugar intake in the UK: a nationally representative cross-sectional study. BMJ Open 9, e027546.

48. Cediel G, Reyes M, da Costa Louzada ML, et al. (2018) Ultraprocessed foods and added sugars in the Chilean diet (2010). Public Health Nutr 21, 125-133.

49. Jacome-Sosa MM \& Parks EJ (2014) Fatty acid sources and their fluxes as they contribute to plasma triglyceride concentrations and fatty liver in humans. Curr Opin Lipidol 25 , 213-220.

50. Softic S, Cohen DE \& Kahn CR (2016) Role of dietary fructose and hepatic de novo lipogenesis in fatty liver disease. Dig Dis Sci 61, 1282-1293.

51. Aune D, Giovannucci E, Boffetta P, et al. (2017) Fruit and vegetable intake and the risk of cardiovascular disease, total cancer and all-cause mortality - a systematic review and dose-response meta-analysis of prospective studies. Int $J$ Epidemiol 46, 1029-1056.

52. Freedman DS, Dietz WH, Srinivasan SR, et al. (1999) The relation of overweight to cardiovascular risk factors among children and adolescents: the Bogalusa Heart Study. Pediatrics 103, $1175-1182$

53. Umer A, Kelley GA, Cottrell LE, et al. (2017) Childhood obesity and adult cardiovascular disease risk factors: a systematic review with meta-analysis. BMC Public Health 17, 683.

54. Fardet A (2016) Minimally processed foods are more satiating and less hyperglycemic than ultra-processed foods: a preliminary study with 98 ready-to-eat foods. Food Funct $\mathbf{7}$, $2338-2346$.

55. Filgueiras AR, Pires de Almeida VB, Koch Nogueira PC, et al. (2019) Exploring the consumption of ultra-processed foods and its association with food addiction in overweight children. Appetite 135, 137-145.

56. Mallarino C, Gómez LF, González-Zapata L, et al. (2013) Advertising of ultra-processed foods and beverages: children as a vulnerable population. Rev Saude Publica $\mathbf{4 7}$, 1006-1010.

57. Pulker CE, Scott JA \& Pollard CM (2018) Ultra-processed family foods in Australia: nutrition claims, health claims and marketing techniques. Public Health Nutr 21, 38-48.

58. Huet F, Roubille C \& Roubille F (2019) Is hypertriglyceridemia atherogenic? Curr Opin Lipidol 30, 291-299.

59. Berenson GS, Srinivasan SR, Cresanta JL, et al. (1981) Dynamic changes of serum lipoproteins in children during adolescence and sexual maturation. Am J Epidemiol 113, 157-170.

60. Birch L, Savage JS \& Ventura A (2007) Influences on the development of children's eating behaviours: from infancy to adolescence. Can J Diet Pract Res 68, s1-s56.

61. Fernandez-Jimenez R, Al-Kazaz M, Jaslow R, et al. (2018) Children present a window of opportunity for promoting health. J Am Coll Cardiol 72, 3310-3319.

62. FAO (2015) Guidelines on the Collection of Information on Food Processing Through Food Consumption Surveys. Rome: FAO.

63. Ministry of Health of Brazil (2014) Dietary Guidelines for the Brazilian Population, 2nd ed. Brasilia: Ministry of Health of Brazil. 